\title{
Highly edge-connected regular graphs without large factorizable subgraphs
}

\author{
Davide Mattiolo, Eckhard Steffen ${ }^{\dagger}$
}

\begin{abstract}
We construct highly edge-connected $r$-regular graph which do not contain $r-2$ pairwise disjoint perfect matchings. The results partially answer a question stated by Thomassen $\lfloor 4$.
\end{abstract}

\section{Introduction}

We consider finite graphs which may have parallel edges but no loops. Let $r \geq 0$ be an integer. A graph $G$ is $r$-regular, if every vertex has degree $r$. For $1 \leq k \leq r$, a graph $H$ is a $k$-factor of $G$, if $H$ is a spanning $k$-regular subgraph of $G$. Recently, Thomassen stated the following problem.

Problem $1.1(|4|)$. Is every $r$-regular $r$-edge-connected graph of even order the union of $r-2$-factors and a 2-factor?

The statement is true for $r=3$. An $r$-regular graph $G$ is an $r$-graph, if $\left|\partial_{G}(S)\right| \geq r$ for every $S \subseteq V(G)$ with $|S|$ odd; $\partial_{G}(S)$ denotes the set of edges with precisely one end in $S$. We write $\partial(S)$ instead of $\partial_{G}(S)$ when it is clear the graph we are considering.

An $r$-graph is poorly matchable, if it does not contain two disjoint 1factors. Clearly, every bridgeless cubic graph with edge chromatic number 4 is poorly matchable. Rizzi $[3\rfloor$ constructed poorly matchable $r$-graphs for each $r \geq 4$. All of them contain an edge of multiplicity $r-2$ and therefore, they have a 4-edge cut. However, the poorly matchable 4-graphs are 4-edgeconnected and therefore, they provide a negative answer to Problem 1.1. The 4-graphs constructed in [1] are also poorly matchable. The following theorem is the main result of this note and it provides a negative answer to the question of Problem 1.1 for every positive integer which is a multiple of 4 .

${ }^{*}$ Department of Physics, Informatics and Mathematics, University of Modena and Reggio Emilia, Via Campi 213/b, 41126 Modena, Italy. Email: davide.mattiolo@unimore.it

${ }^{\dagger}$ Paderborn Center for Advanced Studies and Institute for Mathematics, Paderborn University, Warburger Str. 100, 33098 Paderborn, Germany. Email: es@upb.de 
Theorem 1.2. Let $t, r$ be positive integers and $r \geq 4$. There are infinitely many $t$-edge-connected $r$-graphs which do not contain $r-2$ pairwise disjoint 1-factors, where

- $t=r$, if $r \equiv 0 \bmod 4$;

- $t=r-1$, if $r \equiv 1 \bmod 2$;

- $t=r-2$, if $r \equiv 2 \bmod 4$.

Indeed, we prove that for any $r \geq 4$, there are $r$-graphs of order 60 and simple $r$-graphs of order $70(r-1)$ with this properties.

\section{Proof of Theorem 1.2}

Let $G$ be a graph and $N_{1}, \ldots, N_{k}$ be a collection of subsets of $E(G)$. The graph $G^{\prime}=G+\left(N_{1}+\cdots+N_{k}\right)$ consists of all vertices of $G$, i.e. $V\left(G^{\prime}\right)=V(G)$, and $E\left(G^{\prime}\right)=E(G) \cup \bigcup_{i=1}^{k} N_{i}^{\prime}$, where $N_{i}^{\prime}$ is a copy of $N_{i}$.

\subsection{Perfect matchings of the Petersen graph}

The edge set of a 1-factor of a graph $G$ is a perfect matching of $G$. A collection $\mathcal{C}$ is a set of objects where repetitions are allowed. Namely we can formally define it as a set $C=\left\{C_{1}, \ldots, C_{n}\right\}$ together with a function $m: C \rightarrow \mathbb{N}$ which gives the multiplicity of each object $C_{j}$ in $\mathcal{C}$, that is the number of occurrences of $C_{j}$ in $\mathcal{C}$. A subcollection $\mathcal{C}^{\prime}$ of $\mathcal{C}$ is a subset $C^{\prime} \subseteq C$ with a function $m^{\prime}: C^{\prime} \rightarrow \mathbb{N}$ such that $m^{\prime}\left(C_{j}\right) \leq m\left(C_{j}\right)$ for all $j \in\{1, \ldots, n\}$. In this case we will write $\mathcal{C}^{\prime} \subseteq \mathcal{C}$.

Our construction heavily relies on the properties of the Petersen graph $P$. Let $v_{0} \ldots v_{5}$ and $u_{1} u_{3} u_{5} u_{2} u_{4}$ be the two disjoint 5-cycles of $P$ such that $u_{1} v_{1}, u_{2} v_{2}, u_{3} v_{3}, u_{4} v_{4}, u_{5} v_{5} \in E(P)$. Let $M_{0}=\left\{u_{i} v_{i}: i \in\{1, \ldots, 5\}\right\}$ and let $M_{1}$ be the only other perfect matching containing $u_{1} v_{1}$. Moreover let $M_{i}=\left\{u_{i+1} v_{j+1}: u_{i} v_{j} \in M_{1}\right\}$, where the sum of indices is taken modulo 5 , see Figure 1. Let $\mathcal{M}=\left\{N_{1}, \ldots, N_{k}\right\}$ be a collection of perfect matchings of $P$ and let $P^{\mathcal{M}}=P+\sum_{j=1}^{k} N_{j}$. We say that a perfect matching $N$ of $P^{\mathcal{M}}$ is of type $j$, if $N$ is a copy of $M_{j}$. In this case we write $t(N)=j$. We will use the following results of Rizzi $\mid 3\rfloor$.

Proposition $2.1(|3|)$. The function associating to every pair of perfect matchings $M_{i}, M_{j}$ of $P$ the unique edge $e \in M_{i} \cap M_{j}$ is a bijection.

Lemma $2.2(|3|)$. Consider a perfect matching $M_{j}$ of $P$ and let $P^{\prime}=P+M_{j}$. Furthermore let $N_{1}$ and $N_{2}$ be two disjoint perfect matchings of $P^{\prime}$. Then $j \in\left\{t\left(N_{1}\right), t\left(N_{2}\right)\right\}$. 

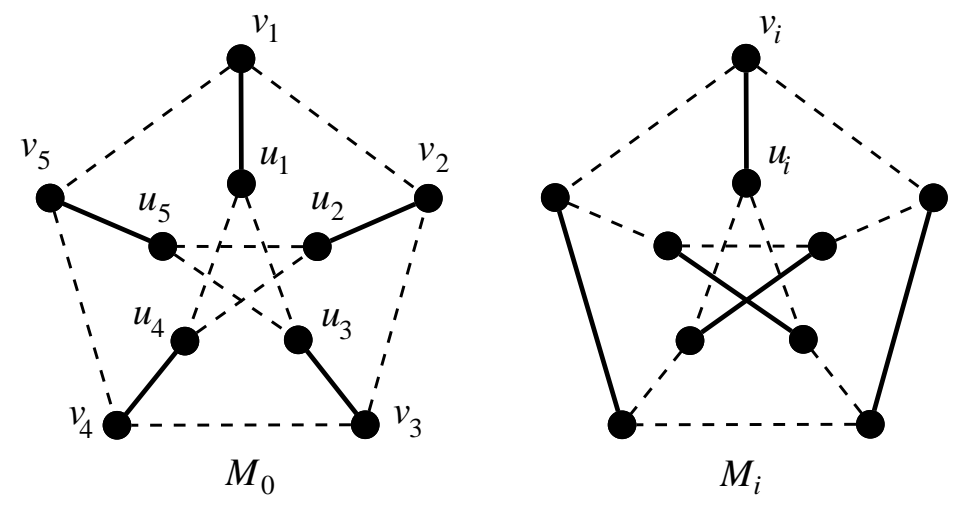

Figure 1: The perfect matchings $M_{0}, \ldots, M_{5}$ of $P$.

Lemma 2.3. Let $\mathcal{M}$ be a collection of $k$ perfect matchings of $P$. If $\mathcal{M}^{\prime}=$ $\left\{M_{1}^{\prime}, \ldots, M_{k}^{\prime}, M_{k+1}^{\prime}\right\}$ is a collection of $k+1$ pairwise disjoint perfect matchings of $P^{\mathcal{M}}$, then $\mathcal{M} \subseteq \mathcal{M}^{\prime}$.

Proof. We argue by induction over $k \in \mathbb{N}$. If $k=1$, then the statement holds by Lemma 2.2. So let $k \geq 2$ and $\mathcal{M}^{\prime}=\left\{M_{1}^{\prime}, \ldots, M_{k+1}^{\prime}, M_{k+1}^{\prime}\right\}$ be pairwise disjoint perfect matchings of $P^{\mathcal{M}}$.

If $M_{i}^{\prime}=M_{j}^{\prime}$ for all $i, j \in\{1, \ldots, k+1\}$, then $\mathcal{M}$ must contain a unique perfect matching repeated $k$ times, and so $\mathcal{M} \subseteq \mathcal{M}^{\prime}$.

Otherwise there are $i, j \in\{1, \ldots, k+1\}$ such that $M_{i}^{\prime} \neq M_{j}^{\prime}$. There is a unique edge $e \in P$ such that $\{e\}=M_{i}^{\prime} \cap M_{j}^{\prime}$. Such an edge must be a multiedge in $P^{\mathcal{M}}$. Then either $M_{i}^{\prime}$ or $M_{j}^{\prime}$ has been added to $P$ in order to obtain $P^{\mathcal{M}}$. This means that both $\mathcal{M}$ and $\mathcal{M}^{\prime}$ contain a copy $M$ of the same perfect matching. Therefore, by the inductive hypothesis, $\mathcal{M} \backslash\{M\} \subseteq \mathcal{M}^{\prime} \backslash\{M\}$, and so $\mathcal{M} \subseteq \mathcal{M}^{\prime}$.

\section{$2.24 k$-edge-connected $4 k$-graphs without $4 k-2$ pairwise dis- joint perfect matchings}

For $k \geq 1$, let $P_{k}=P+k M_{0}+(k-1)\left(M_{1}+M_{3}+M_{4}\right)$.

Lemma 2.4. For all $k \geq 1: P_{k}$ is $4 k$-edge-connected and $4 k$-regular.

Proof. By definition, $P_{k}$ is $4 k$-regular. Let $X \subseteq V(P)$. If $|X|$ is odd, then every perfect matching intersects $\partial(X)$. Hence, $|\partial(X)| \geq 3+k+3(k-1)=4 k$. If $|X|$ is even, then it suffices to consider the cases $|X| \in\{2,4\}$. Since $P$ has girth 5 , the subgraph induced by $X$ is a path $P_{X}$ on either 2 or 4 vertices, having some multiple edge. In both cases, since the maximum multiplicity of an edge is $2 k$, we have that $\partial(X)$ contains $2 k$ edges per both end vertices of $P_{X}$, namely $|\partial(X)| \geq 4 k$. 


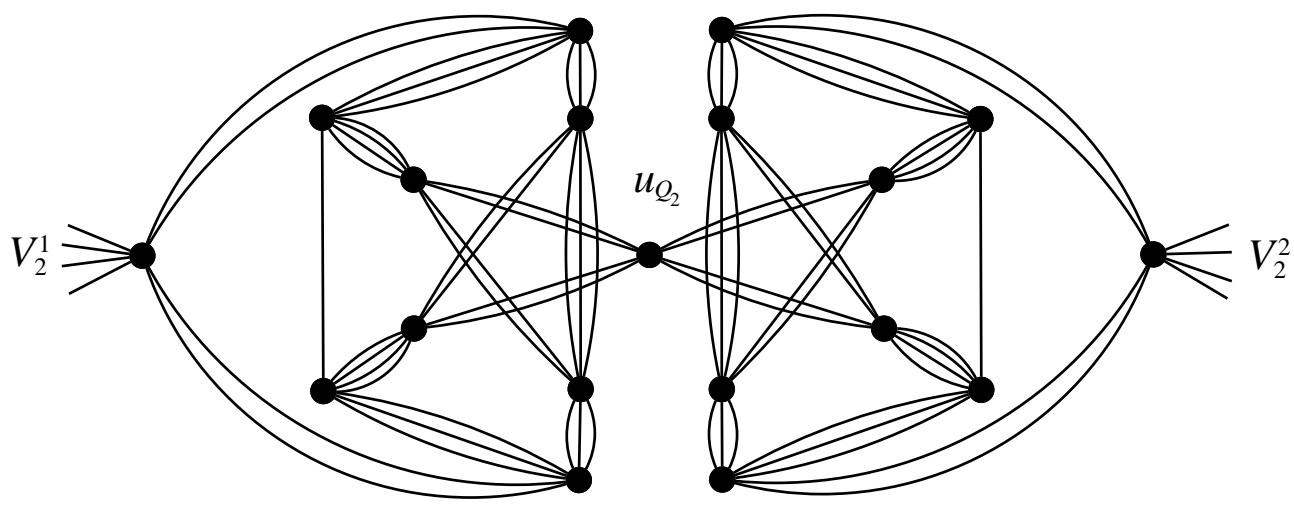

Figure 2: The subgraph $Q_{2}$.

Consider two copies $P_{k}^{1}$ and $P_{k}^{2}$ of $P_{k}$. If $u$ is a vertex (or edge) of $P_{k}$, then we denote $u^{i}$ the corresponding vertex (or edge) inside $P_{k}^{i}$. For $i \in\{1,2\}$ remove the multiedge $u_{1}^{i} v_{1}^{i}$ from $P_{k}^{i}$ and let $Q_{k}$ be the graph obtained by identifying $u_{1}^{1}$ and $u_{1}^{2}$ to the (new) vertex $u_{Q_{k}}$. It holds $d_{Q_{k}}\left(u_{Q_{k}}\right)=4 k$, and the $2 k$ edges of $\partial\left(u_{Q_{k}}\right)$ which are incident to vertices of $V\left(P_{k}^{i}\right)$ are denoted by $U_{k}^{i}$. If $Q_{k}$ is a subgraph of a graph $G$, then let $V_{k}^{i}=\left\{x v_{1}^{i} \in E(G): x \notin\right.$ $\left.V\left(Q_{k}\right)\right\}$.

Let $\left\{N_{1}, \ldots, N_{n}\right\}$ be a collection of perfect matchings of a graph $G$, define the function $\phi: E(G) \rightarrow \mathbb{Z}_{2}^{n}, e \mapsto\left(\phi_{1}(e), \ldots, \phi_{n}(e)\right)$ such that

$$
\phi_{j}(e)= \begin{cases}1 & \text { if } e \in N_{j} \\ 0 & \text { otherwise }\end{cases}
$$

Moreover, if $W \subseteq E(G)$, then let $\phi(W)=\sum_{e \in W} \phi(e)$. For a vector $x=$ $\left(x_{1}, \ldots, x_{n}\right) \in \mathbb{Z}_{2}^{n}$ the number of its non-zero entries is denoted by $\omega(x)$.

Lemma 2.5. Let $Q_{k}$ be a subgraph of a graph $G$ with $\partial\left(V\left(Q_{k}\right)\right)=V_{k}^{1} \cup V_{k}^{2}$. If, for all $i \in\{1,2\}, d_{G}\left(v_{1}^{i}\right)=4 k$ and $\mathcal{N}=\left\{N_{1}, \ldots, N_{4 k-2}\right\}$ is a family of pairwise disjoint perfect matchings of $G$, then

$$
\omega\left(\phi\left(V_{k}^{1}\right)\right)=\omega\left(\phi\left(V_{k}^{2}\right)\right)=2 k-1 .
$$

Proof. Every perfect matching of $G$ intersects $\partial\left(V\left(Q_{k}\right)\right)$ precisely once since $\left|V\left(Q_{k}\right)\right|$ is odd.

It remains to show that $V_{k}^{i}$ intersects precisely $2 k-1$ elements of $\mathcal{N}$. Recall that $Q_{k}$ is constructed by using two copies of $P+\sum_{M \in \mathcal{M}} M$, where

$$
\mathcal{M}=\{M_{0}, \underbrace{M_{0}, M_{1}, M_{3}, M_{4}, \ldots, M_{0}, M_{1}, M_{3}, M_{4}}_{(k-1) \text {-times }}\} .
$$

Since $\left|V_{k}^{i}\right|=2 k$ and $\mathcal{N}$ contains $4 k-2$ perfect matchings, it follows that $\omega\left(\phi\left(V_{k}^{i}\right)\right) \in\{2 k-2,2 k-1,2 k\}$. Suppose to the contrary that $\omega\left(\phi\left(V_{k}^{1}\right)\right)=$ 


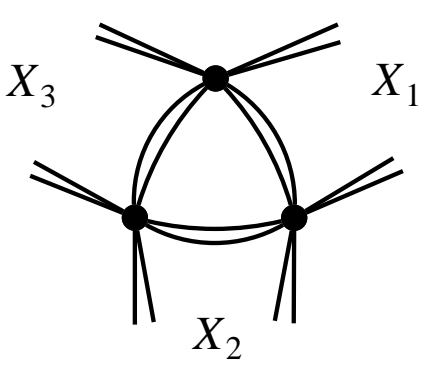

Figure 3: The subgraph $T_{2}$.

$2 k-2$, which is equivalent to $\omega\left(\phi\left(V_{k}^{2}\right)\right)=2 k$. Furthermore, $U_{k}^{1}\left(U_{k}^{2}\right)$ intersects the same matchings of $\mathcal{N}$ as $V_{k}^{2}\left(V_{k}^{1}\right)$. Therefore, there is a family $\mathcal{N}_{P}$ of $4 k-2$ pairwise disjoint perfect matching in $P_{k}^{1}$ such that $\mathcal{M} \nsubseteq \mathcal{N}_{P}$, a contradiction to Lemma 2.3. Hence, $\omega\left(\phi\left(V_{k}^{1}\right)\right)=\omega\left(\phi\left(V_{k}^{2}\right)\right)=2 k-1$.

Let $T_{k}$ be the graph on three vertices $x_{1}, x_{2}, x_{3}$ such that, for all $i \neq j$, there are $k$ parallel edges connecting $x_{i}$ to $x_{j}$.

Let $G$ be a cubic graph. Construct the graph $S_{k}(G)$ as follows: replace every node $v \in V(G)$ by a copy $T_{k}^{v}$ of the graph $T_{k}$ and every edge $e \in E(G)$ by a copy $Q_{k}^{e}$ of the graph $Q_{k}$. If the vertex $v^{\prime}$ is adjacent with the edge $e^{\prime}$, then the graphs $T_{k}^{v^{\prime}}$ and $Q_{k}^{e^{\prime}}$ are connected by $2 k$ edges. More precisely, add $k$ edges connecting $v_{1}^{1}$ (or $v_{1}^{2}$ ) together with $x_{i}$ and $k$ edges connecting $v_{1}^{1}$ (or $v_{1}^{2}$ ) together with $x_{i+1}$, for suitable $i \in \mathbb{Z}_{3}$. Connect those graphs in such a way that the resulting graph $S_{k}(G)$ is $4 k$-regular.

Let $p=w_{1} e_{1} \ldots w_{n} e_{n}$ be a path in $G$, for $w_{j} \in V(G)$ and $e_{j} \in E(G)$, then the chain $C=T_{k}^{w_{1}} Q_{k}^{e_{1}} \ldots T_{k}^{w_{n}} Q_{k}^{e_{n}}$ consists of graphs which are connected to the previous and the next one, with respect to the chain order, in $S_{k}(G)$. In this case, we will say that the chain of a graph $C$ forms a path in $S_{k}(G)$.

Lemma 2.6. Let $G$ be a bridgeless cubic graph. For all $k \geq 1: S_{k}(G)$ is a $4 k$-edge-connected $4 k$-regular graph.

Proof. $S_{k}(G)$ is $4 k$-regular by construction. We show that there are $4 k$ pairwise disjoint paths between any two vertices of $S_{k}(G)$. Consider the graph $R_{k}=P_{k}-u_{1} v_{1}$, where we remove from $P_{k}$ all $(2 k)$ edges connecting $u_{1}$ to $v_{1}$.

Claim 1. The following statements hold:

i. there are $2 k$ edge-disjoint $u_{1} v_{1}$-paths in $R_{k}$;

ii. for all $w \in V\left(P_{k}\right) \backslash\left\{u_{1}, v_{1}\right\}$ there are $2 k w u_{1}$-paths and $2 k$ wv $v_{1}$-paths which are pairwise edge-disjoint in $R_{k}$;

iii. for all $w_{1} \neq w_{2} \in V\left(P_{k}\right) \backslash\left\{u_{1}, v_{1}\right\}$, there exists $t \in\{0,1, \ldots, 2 k\}$ such that 
- there are $t$ edge-disjoint $w_{1} w_{2}$-paths containing $u_{1} v_{1}$ in $P_{k}$;

- there are $4 k-t$ edge-disjoint $w_{1} w_{2}$-paths in $R_{k}$, which are moreover edge-disjoint from the previous ones.

$i v$. for all $x_{i} \neq x_{j} \in V\left(T_{k}\right)$, there are $2 k$ edge-disjoint $x_{i} x_{j}$-paths in $T_{k}$.

Proof. By Lemma 2.4 there are $4 k$ edge-disjoint $u_{1} v_{1}$-paths in $P_{k}$. Since $\mu\left(u_{1} v_{1}\right)=2 k$ there are $2 k$ edge-disjoint $u_{1} v_{1}$-paths in $R_{k}$ and $i$. is proved.

Let $w \in V\left(P_{k}\right) \backslash\left\{u_{1}, v_{1}\right\}$. By Lemma 2.4, there are $4 k$ edge-disjoint $w v_{1^{-}}$ paths in $P_{k}$. Then, since $P_{k}$ is $4 k$-regular and $u_{1} v_{1}$ is an edge of multiplicity $\mu\left(u_{1} v_{1}\right)=2 k$, there are $2 k$ of those paths ending with the edge $u_{1} v_{1}$. Thus, there are $2 k w u_{1}$-paths and $2 k w v_{1}$-paths which are pairwise edge-disjoint in $R_{k}$ and so $i i$. is proved.

In order to prove statement iii., pick two different vertices $w_{1}$ and $w_{2}$ in $V\left(P_{k}\right) \backslash\left\{u_{1}, v_{1}\right\}$. Since $P_{k}$ is $4 k$-edge-connected there are $4 k$-edge-disjoint $w_{1} w_{2}$-paths in $P_{k}$. Let $t$ be the number of such paths containing the edge $u_{1} v_{1}$. Then $t \leq \mu\left(u_{1} v_{1}\right)=2 k$.

The last statement holds since there are $k$ pairwise edge-disjoint paths $x_{i} x_{j}$ and there are $k$ pairwise edge-disjoint paths $x_{i} x_{t} x_{j}$, for $t \neq i, j$. Thus, the claim is proved.

Let $y_{1} \neq y_{2} \in V\left(S_{k}(G)\right)$. There are copies of $T_{k}$ or $Q_{k}$, say $Y_{1}, Y_{2}$, such that $y_{i} \in Y_{i}$.

Case 1: $Y_{1}$ and $Y_{2}$ correspond to two vertices $w_{1}$ and $w_{2}$ of $G$, that is, they both are copies of $T_{k}$. If $w_{1}=w_{2}$, then $Y_{1}=Y_{2}$ and the statement is trivial. If $w_{1} \neq w_{2}$, since $G$ is bridgeless, there are two edge-disjoint $w_{1} w_{2}$-paths in $G$. These paths correspond to two chains of (internally) different subgraphs $C=Y_{1} N_{1} \ldots N_{p} Y_{2}$ and $C^{\prime}=Y_{1} N_{1}^{\prime} \ldots N_{q}^{\prime} Y_{2}$ that both form a path in $S_{k}(G)$. Let $s_{j}, t_{j}$ the nodes of $N_{j}$ which are adjacent to $N_{j-1}$ and $N_{j+1}$ respectively. Let $s_{1}$ be adjacent to $Y_{1}$ and $t_{p}$ be adjacent to $Y_{2}$. Define in the very same way the vertices $s_{j}^{\prime}, t_{j}^{\prime}$ in $C^{\prime}$. By Claim 1 , there are $2 k$ pairwise edge-disjoint $s_{1} t_{p}$-paths in $C$ and $2 k$ pairwise edge-disjoint $s_{1}^{\prime} t_{q}^{\prime}$-paths in $C^{\prime}$. Notice that $s_{1} \neq s_{1}^{\prime}$ and $t_{p} \neq t_{q}^{\prime}$. By Claim 1 , there are $2 k$ pairwise edge-disjoint $s_{1} s_{1}^{\prime}$ paths passing through $Y_{1}$ and $2 k$ edge-disjoint $t_{p} t_{q}^{\prime}$-paths passing through $Y_{2}$. Therefore, all these paths combine to $4 k$ edge-disjoint $y_{1} y_{2}$-paths in $S_{k}(G)$.

Case 2: If $Y_{1}$ and $Y_{2}$ correspond to a vertex and an edge, or to two edges of $G$, say $a_{1}$ and $a_{2}$, then there is a circuit in $G$ which contains $a_{1}$ and $a_{2}$. By a similar argumentation as above we deduce that there are $4 k$-edge-disjoint $y_{1} y_{2}$-paths in $S_{k}(G)$.

Theorem 2.7. Let $G$ be a bridgeless cubic graph with an even number of edges. For all $k \geq 1: S_{k}(G)$ is a $4 k$-edge-connected $4 k$-graph without $4 k-2$ pairwise disjoint perfect matchings.

Proof. By Lemma 2.6, $S_{k}(G)$ is $4 k$-edge-connected, $4 k$-regular and it holds $\left|V\left(S_{k}(G)\right)\right|=19|E(G)|+3|V(G)| \equiv 0 \bmod 2$. Thus, $S_{k}(G)$ is a $4 k$-graph. 
Suppose to the contrary that $S_{k}(G)$ has $4 k-2$ pairwise disjoint perfect matchings. Consider a vertex $v \in V(G)$ and the corresponding subgraph $T_{k}^{v}$. Since $T_{k}^{v}$ has three vertices it follows that no component of $\phi\left(\partial_{S_{k}(G)}\left(V\left(T_{k}^{v}\right)\right)\right)$ is 0 . Let $\partial_{G}(v)=\left\{e_{1}, e_{2}, e_{3}\right\}$ and $X_{i}$ be the set of $2 k$ edges connecting $T_{k}^{v}$ to $Q_{k}^{e_{i}}$, see Figure 3. By Lemma 2.5, for all $i \in\{1,2,3\}$, we have that $\omega\left(\phi\left(X_{i}\right)\right)=2 k-1$. Since the cardinality of the symmetric difference of three odd sets is odd it follows that there is a $j \in\{1,2, \ldots, 4 k-2\}$ such that $0=\sum_{i=1}^{3} \phi_{j}\left(X_{i}\right)=\phi_{j}\left(\partial_{S_{k}(G)}\left(V\left(T_{k}^{v}\right)\right)\right)$, a contradiction.

\subsection{Highly connected $r$-graphs on 60 vertices}

To prove the other cases of Theorem 1.2, we continue with the construction of regular graphs on 60 vertices.

For $k \geq 1$ : Let $H_{k}$ be the graph which is obtained from three copies $Q_{k}^{1}, Q_{k}^{2}, Q_{k}^{3}$ of $Q_{k}$. In order to simplify the description let $z_{j}$ be the vertex $v_{1}^{j}$ of $Q_{k}$, for $j \in\{1,2\}$. For $i \in\{1,2,3\}$, if $u$ is a vertex of $Q_{k}$ we denote by $u^{i}$ the corresponding vertex of the copy $Q_{k}^{i}$. Glue them together with the graph $T_{k}$ as follows: for all $i \in\{1,2,3\}$,

- add $k$ edges connecting $x_{i+1}$ of $T_{k}$ to $z_{1}^{i}$ of $Q_{k}^{i}$;

- add $k$ edges connecting $x_{i+2}$ of $T_{k}$ to $z_{1}^{i}$ of $Q_{k}^{i}$;

- add $k$ edges connecting $z_{2}^{i}$ of $Q_{k}^{i}$ to $z_{2}^{i+1}$ of $Q_{k}^{i+1}$;

where the indices are added modulo 3. The graph $\mathrm{H}_{2}$ is depicted in Figure 4 .

Lemma 2.8. For all $k \geq 1: H_{k}$ is a $4 k$-edge-connected $4 k$-graph of order 60 without $4 k-2$ pairwise disjoint perfect matchings.

Proof. Let $K_{2}^{3}$ be the unique (loopless) cubic graph on two vertices. $H_{k}$ is obtained from $S_{k}\left(K_{2}^{3}\right)$ by removing one $T_{k}$ and then connecting the vertices of degree $2 k$ pairwise by $k$ (parallel) edges. Clearly, $H_{k}$ is $4 k$-regular. Note that $\left\{z_{2}^{1}, z_{2}^{2}, z_{2}^{3}\right\}$ induce a triangle $T$ in $H_{k}$ where any two vertices are connected by $k$ edges. Furthermore, for any $2 k$ pairwise edge-disjoint paths which connect two vertices of $\left\{z_{2}^{1}, z_{2}^{2}, z_{2}^{3}\right\}$ in $S_{k}\left(K_{2}^{3}\right)$ and do not contain any edge of $S_{k}\left(K_{2}^{3}\right)-T_{k}$ there are $2 k$ corresponding paths in $H_{k}$. Hence, $H_{k}$ is $4 k$ edge-connected.

Suppose to the contrary that $H_{k}$ has $4 k-2$ pairwise disjoint perfect matchings $\mathcal{N}=\left\{N_{1}, \ldots, N_{4 k-2}\right\}$. By Lemma 2.5, for each $i \in\{1,2,3\}$, there are $2 k-1$ edges of $E(T)$ which intersect an element of $\mathcal{N}$ and which are incident to $z_{2}^{i}$, a contradiction.

Next we will identify 4 pairwise disjoint matchings in $H_{2}$. These matchings will be used to complete the proof of Theorem 1.2. 


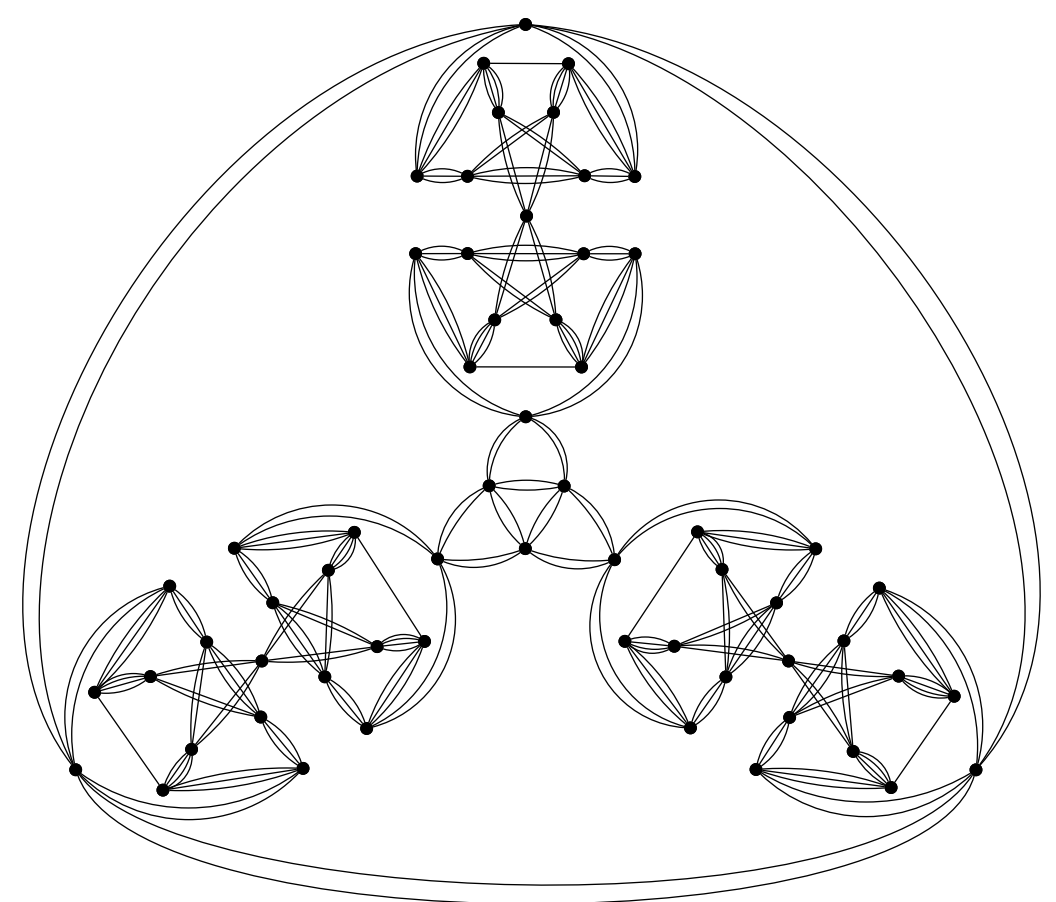

Figure 4: $\mathrm{H}_{2}$ is an 8-edge-connected 8-graph on 60 vertices without 6 pairwise disjoint perfect matchings.

Consider a copy of $Q_{k}$ inside a graph $G$, such that both $V_{k}^{1}$ and $V_{k}^{2}$ are non-empty. Let $M$ be a perfect matching of $G$. Then w.l.o.g. $\left|V_{k}^{1} \cap M\right|=1$ and $\left|V_{k}^{2} \cap M\right|=0$. The unique perfect matching in $P_{k}=P_{k}^{1}+2 k u_{1}^{1} v_{1}^{1}$ containing the edges of $M \cap E\left(P_{k}^{1}\right)$ is of type 0 or 1 , suppose of type 0 . In the same way, the unique perfect matching in $P_{k}=P_{k}^{2}+2 k u_{1}^{2} v_{1}^{2}$ containing the edges of $M \cap E\left(P_{k}^{2}\right)$ is of type 3 or 4 , suppose of type 3 . In this case we say that $Q_{k}$ is of type $(0,3)$. For example, the bold perfect matching depicted in Figure 5 is such that all $Q_{k}^{i}$ s are of type $(0,4)$. We call $N_{0}$ such a perfect matching in $H_{k}$. Moreover, for $i \in\{1,2,3\}$, let $N_{i}$ be the perfect matching of $H_{k}$ such that:

- $Q_{k}^{i}$ is of type $(1,3)$;

- $Q_{k}^{i+1}$ is of type $(3,0)$;

- $Q_{k}^{i+2}$ is of type $(4,1)$;

where sums of indices are taken modulo 3 . In Figure $5 N_{1}$ is depicted using normal lines, $N_{2}$ is depicted using dotted lines and $N_{3}$ is depicted using dashed lines.

By construction of the perfect matchings $N_{0}, N_{1}, N_{2}, N_{3}$, the following lemma, which will be needed for the proof of Theorem 1.2, follows. 


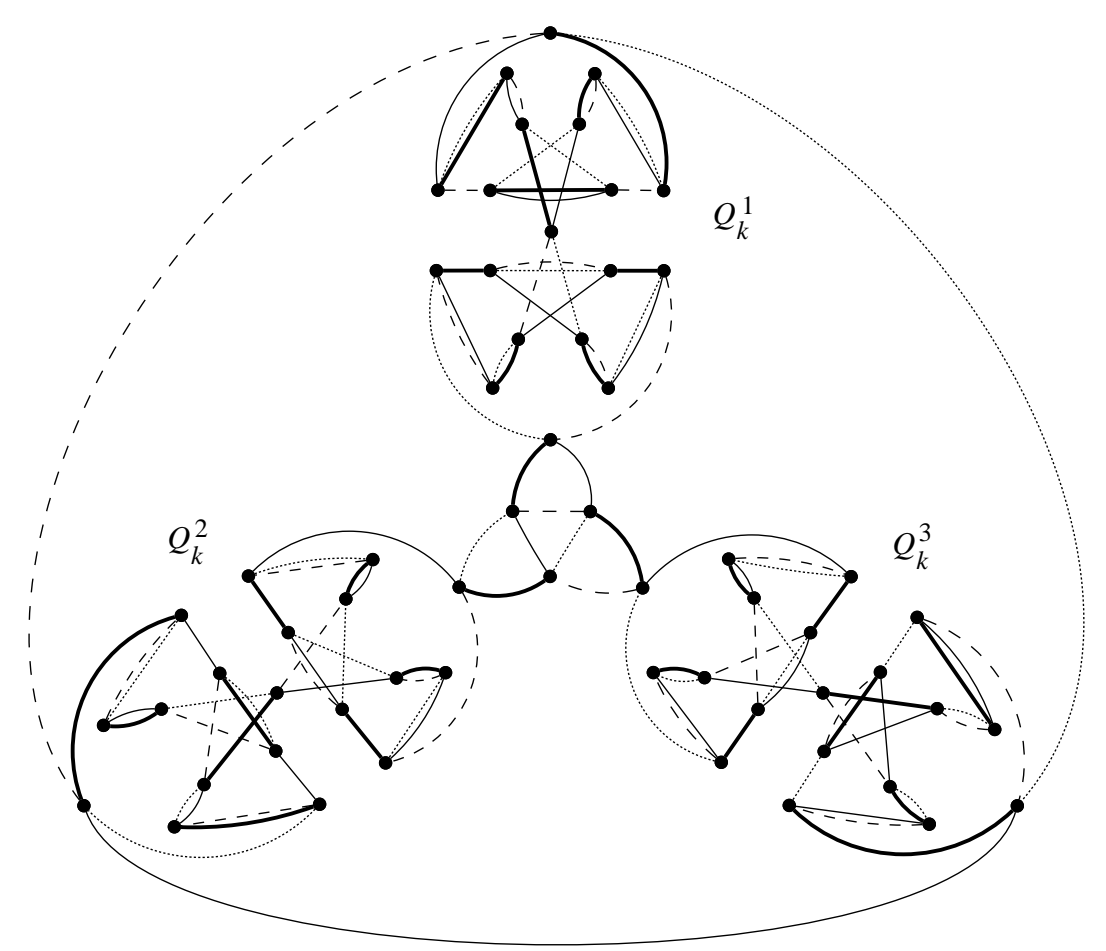

Figure 5: Four pairwise disjoint perfect matchings in $H_{k}$.

Lemma 2.9. For all $k \geq 1: H_{k+1}=H_{k}+\left(N_{0}+N_{1}+N_{2}+N_{3}\right)$.

Lemma 2.10. For all $t \geq 1$, there is a $2 t$-edge-connected $(2 t+1)$-graph on 60 vertices without $2 t-1$ pairwise disjoint perfect matchings.

Proof. Case 1: $t=2 k+1$ for a $k \geq 1$. Let $H_{k}^{\prime}=H_{k}+\left(N_{0}+N_{1}+N_{2}\right)$. Since the graph $\tilde{H}=H_{k}\left[N_{0}+N_{1}+N_{2}\right]$ is a 3-edge-colorable connected cubic graph, we have that for all $X \subseteq V(\tilde{H}),\left|\partial_{\tilde{H}}(X)\right| \geq 3$, if $X$ is odd and $\left|\partial_{\tilde{H}}(X)\right| \geq 2$, if $X$ is even. Then $H_{k}^{\prime}$ is $(4 k+2)$-edge-connected $(4 k+3)$-graph. From the equality $H_{k}^{\prime}=H_{k+1}-N_{3}$ we deduce that it has no $4 k+1$ pairwise disjoint perfect matchings.

Case 2: $t=2 k$ for a $k \geq 1$. The graph $H_{k}^{\prime \prime}=H_{k}+N_{0}$ is a $4 k$-edgeconnected $(4 k+1)$-graph. Since $H_{k}^{\prime \prime}=H_{k+1}-\left(N_{1}+N_{2}+N_{3}\right)$, it follows that $H_{k}^{\prime \prime}$ has no $4 k-1$ pairwise disjoint perfect matchings.

Lemma 2.11. For all $k \geq 1$, there is a $4 k$-edge-connected $(4 k+2)$-graph on 60 vertices without $4 k$ pairwise disjoint perfect matchings.

Proof. The graph $H_{k}^{\prime \prime \prime}=H_{k}+N_{0}+N_{1}$ is a $4 k$-edge-connected $(4 k+2)$-graph. It has no $4 k$ pairwise disjoint perfect matchings because $H_{k}^{\prime \prime \prime}=H_{k+1}-\left(N_{2}+\right.$ $\left.N_{3}\right)$. 
Theorem 1.2 now follows from Lemmas 2.8, 2.10, and 2.11. It remains to construct simple graphs with the desired property and to show how to expand vertices.

\subsection{Simple graphs}

Let $v$ be a vertex of a graph $G$ such that $d_{G}(v)=t$. Moreover let $v_{1}, \ldots, v_{t}$ be the not necessarily distinct neighbors of $v$ and $u_{1}, \ldots, u_{t}$ be the vertices of degree $t-1$ of $K_{t, t-1}$. The Meredith extension [2] applied to $G$ at $v$ produces the graph $G_{v}$ obtained from $G-v$ and a copy of the complete graph $K_{t, t-1}$ by adding all edges $v_{i} u_{i}$, for $i \in\{1, \ldots, t\}$. Notice that $G$ is $t$-edge-connected if and only if $G_{v}$ is $t$-edge-connected. Furthermore, it is easy to see that for $t \geq 2, G$ does not have $t$ pairwise disjoint perfect matchings if and only if $G_{v}$ does not have $t$ pairwise disjoint perfect matchings.

Let $\mathcal{V} \subset V\left(H_{k}\right)$ be a vertex cover of $H_{k}$. If Meredith extension is applied on every vertex of $\mathcal{V}$, then we obtain simple $r$-edge-connected $r$-graphs without $r-2$ pairwise disjoint perfect matchings. In particular, there is a vertex cover $\mathcal{V}$ of $H_{k}$ such that $|\mathcal{V}|=35$. Thus, expanding the vertices of $\mathcal{V}$ at the graphs of Lemmas 2.8, 2.10, and 2.11 yields simple $t$-edge-connected $r$-graphs of order $70(r-1)$ with the desired properties. Repeated application of Meredith extension yields infinite families of such graphs.

\section{References}

[1] G. Mazzuoccolo, An upper bound for the excessive index of an r-graph, J. Graph Theory, 73 (2013), 377-385.

[2] G. H. J. Meredith, Regular $n$-valent $n$-connected non-hamiltonian non$n$-edge colorable graphs, J. Comb. Theory Ser. B, 14 (1973), 55-60.

[3] R. Rizzi, Indecomposable $r$-graphs and some other counterexamples, J. Graph Theory, 32 (1999), 1-15.

[4] C. Thomassen, Factorizing regular graphs, J. Comb. Theory Ser. B (2019), https://doi.org/10.1016/j.jctb.2019.05.002 (article in press) 